\title{
Metacaspases are caspases. Doubt no more
}

\author{
D Carmona-Gutierrez ${ }^{1}$, K-U Fröhlich ${ }^{1}$, G Kroemer ${ }^{2,3,4}$ and F Madeo ${ }^{\star, 1}$ \\ Cell Death and Differentiation (2010) 17, 377-378; doi:10.1038/cdd.2009.198
}

Caspases are cysteine proteases that cleave their substrates after an aspartate residue. Besides their multiple vital roles ranging from immune regulation to spermatogenesis, they are crucial in most cell death pathways, representing a sort of executing sword in the hands of apoptosis. In 2000, a psi-Blast in-silico approach led Uren et al. ${ }^{1}$ to identify two novel caspase-related families: metacaspases and paracaspases. Paracaspases are involved in the development of MALT lymphoma, but not in cell death execution, and are found both in eukaryotes owning caspases (animals), as well as in organisms lacking caspases. Metacaspases, on the other hand, are found only in eukaryotes that are devoid of caspases, for example, plants, protists and fungi. Similar to caspases, they contain a caspase-specific catalytic diad of histidine and cysteine, as well as a caspase-like secondary structure.

Our lab was among the first to perform experiments on metacaspases showing that the sole metacaspase encoded by the genome of Saccharomyces cerevisiae (which we termed $Y C A 1$ ) is involved in oxidative stress-induced cell death. $^{2}$ Overexpression of $Y C A 1$ caused a type of cell death that was accompanied by apoptotic markers, while deletion of YCA1 protected against apoptosis caused by reactive oxygen species or chronological aging. ${ }^{2}$ We thus had revealed that one metacaspase, YCA1, was involved in the same process as caspases, namely programmed cell death (PCD). Indeed, many groups subsequently unfolded the crucial contribution of metacaspases to cell death execution upon various stresses in yeast and other fungi, ${ }^{3-6}$ as well as in plants. ${ }^{7}$ Bozhkov, Zhivotovsky and colleagues ${ }^{8}$ could even demonstrate that the plant metacaspase mcll-Pa shapes the embryo of Norway spruce (Picea abies) during development, presumably through its implication in developmental cell death. Together with the unequivocal fact of a common evolutionary origin, these results made us conclude that, in functional terms, metacaspases behave like caspases, thus unchaining a stormy scientific debate.

Driven by the fact that caspases occur in animals but metacaspases are present in all kingdoms except animals, many authors deduced that, although cell death is a universal and fundamental process, the implication of caspases in lethal processes is not necessarily conserved. The discovery that metacaspases have a different cleavage specificity than caspases - they hydrolyze proteins after arginine or lysine (basic residues), not after aspartate (an acidic residue) ${ }^{7,9}$ added further fuel to the discussion. In fact, the exclusion of metacaspases from the caspase family and their regrouping into a separate family in the CD clan of cysteine peptidases was demanded. ${ }^{10}$ In other words, it was implicated that metacaspases are not caspases. Nevertheless, we strongly felt that Yca1p, a protein that possesses sequence homology to human caspases, and the knockout of which rescues roughly $40 \%$ of all cell death scenarios tested in yeast, should retain the name 'metacaspase'. Nomenclature reaches beyond particular points of divergence (such as localization, structural features or, as it is for metacaspases, the amino acid specificity) when it refers to functional groups. For instance, we use the expression 'nucleic acids' for DNA or RNA from prokaryotes that by definition lack nuclei. Similarly, we talk about 'mitochondrial DNA' even though this pool of DNA is located in the mitochondria, not in the nucleus.

Admittedly, the question whether metacaspases are caspases (or not) can only be answered by enzymology rather than by the vague comparison of their implication in various lethal signaling pathways. Do caspases and metacaspases cleave similar death-related substrates? We reasoned that, although a major divergence of the amino acid specificity of caspases and metacaspases may have occurred during evolution, their target proteins should fall into similar functional groups if the role of caspases and metacaspases was conserved. Hence, do the degradomes of caspases and metacaspases overlap?

In a recent paper published in Nature Cell Biology, ${ }^{11}$ Peter Bozhkov and colleagues identified the first metacaspase substrate, which they showed to be a functional substrate of mammalian caspase- 3 as well. In their seminal study, they demonstrated that both caspases and metacaspases cleave the phylogenetically conserved protein TSN (Tudor staphylococcal nuclease). The authors revealed that TSN from $P$. abies (PaTSN) is cleaved by its metacaspase (mcll-Pa) at four different sites. This process was blocked either by adding a metacaspase inhibitor or by mutation of a catalytic cysteine. Importantly, PaTSN was shown to be a component of the degradome during both stress-induced and developmental PCD. During embryogenesis, metacaspase activity correlated with proteolysis of endogenous PaTSN in the different embryo stages (high in early and low in mature embryos). Consistently, knockdown of mcll-Pa via

\footnotetext{
${ }^{1}$ Institute of Molecular Biosciences, Karl-Franzens University, Graz, Austria; ${ }^{2}$ INSERM, U848, Villejuif, France; ${ }^{3}$ Institut Gustave Roussy, Villejuif, France and ${ }^{4}$ Université Paris Sud, Paris 11, Villejuif, France

${ }^{*}$ Corresponding author: F Madeo, Institute of Molecular Biosciences (IMB), Karl-Franzens University, Humboldtstrasse 50, 8010 Graz, Austria. Tel: + 433163808878 ; Fax: + 43316380 9898; E-mail: frank.madeo@ uni-graz.at
} 
RNAi resulted in reduced PaTSN cleavage, depending on the remaining metacaspase activity. The authors addressed the physiological significance of TSN cleavage by demonstrating that PaTSN has ribonuclease activity, which was impaired by metacaspase-mediated cleavage during PCD. Moreover, this TSN ribonuclease activity was shown to be essential for plant viability and reproduction. Downregulation of this activity in $P$. abies using a specific inhibitor compromised embryogenesis. In addition, reverse genetics analyses carried out in Arabidopsis by crossing two transfer DNA knockout alleles for each of the two Arabidopsis TSN genes showed impairment of plant fertility and of embryogenesis (including a high incidence of reduced viable pollen and aborted ovule development). ${ }^{11}$

In a further twist, Bozhkov and colleagues ${ }^{11}$ revealed that TSN is involved in human PCD. Just as PaTSN is a substrate for the metacaspase mcll-Pa, human TSN (HsTSN), known to be a multifunctional regulator of gene expression, was cleaved by caspase-3 in vitro and in vivo. Indeed, either mutation of the cleavage site or caspase inhibition prevented the apoptotic proteolysis of HsTSN. Using a non-cleavable HsTSN variant, the authors demonstrated how elevated levels of HsTSN enhance cell proliferation under normal conditions and increase viability upon apoptosis induction. Conversely, depletion of HsTSN by siRNA sufficed to promote apoptosis in human cells. Furthermore, caspase-3-dependent cleavage of HsTSN inhibited its ribonuclease activity and impaired its stimulatory function in pre-mRNA splicing. ${ }^{11}$

The aforementioned experiments nicely illustrate how caspases and metacaspases can degrade similar deathrelevant proteins across phyla. However, differences certainly exist in the TSN-metacaspase/caspase system between plants and animals. Thus, the subcellular localization of TNS or the fragments resulting from its cleavage are not the same. A crucial problem that must be solved in the near future concerns the mechanisms through which metacaspases are activated. For instance, it will be important to know whether metacaspases are activated by an apoptosome-like structure, perhaps as a result of mitochondrial membrane permeabilization (with cytochrome $c$ release?).

Irrespective of the above, Sundström et al. establish that, beyond phylogenetic distance and differences in their proteolytic characteristics, caspases and metacaspases do share natural, death-related substrates. Incredibly enough, they validate the exploration of metacaspase degradomes (in plants and fungi) to identify new substrates relevant for mammalian cell death execution. These findings not only underline the common evolutionary origin of caspases and metacaspases, which is undisputable, but also resolve the open question of functional homology: doubt no more. Caspases and metacaspases do fulfill the criteria of homology (common cellular program, PCD; common substrates, at least in part) and represent variants of the same enzyme that has varied in evolution, in particular regarding the cleavage site. Indicative of this is the mutual exclusion of metacaspases and caspases in the organismal landscape: whereas metacaspases exist in virtually all eukaryotic organisms lacking caspases, the presence of caspases excludes that of metacaspases.

In this light, caspases and their aspartate specificity may constitute a secondary development in just one branch of eukaryotes that includes higher animals. Thus, different lines of evidence reenforce the rational concept that caspases evolved from metacaspases rather than just falling from the sky.

\section{Conflict of interest}

The authors declare no conflict of interest.

Acknowledgements. We are grateful to the Austrian Science Fund FWF (Austria) for grant S-9304-B05 and grant 'Lipotox' to FM and DC-G, grant S-9303B05 to K-UF, as well as to the German Science Association DFG (Germany) for grant MA2587 to FM and DC-G. GK is supported by the Ligue Nationale contre le Cancer (Equipe labellisée), Agence Nationale pour la Recherche (ANR), European Commission (Apo-Sys, ChemoRes, ApopTrain), Fondation pour la Recherche Médicale (FRM), Institut National du Cancer (INCa) and Cancéropôle lle-de-France.

\footnotetext{
1. Uren AG et al. Mol Cell 2000; 6: 961-967.

2. Madeo F et al. Mol Cell 2002; 9: 911-917.

3. Guerin R et al. PLoS One 2009; 4: e6244.

4. Mazzoni C et al. EMBO Rep 2005; 6: 1076-1081.

5. Reiter J et al. J Cell Biol 2005; 168: 353-358.

6. Weinberger M et al. J Cell Sci 2005; 118 (Pt 15): 3543-3553

7. Watanabe N et al. J Biol Chem 2005; 280: 14691-14699.

8. Suarez MF et al. Curr Biol 2004; 14: R339-R340.

9. Vercammen D et al. J Biol Chem 2004; 279: 45329-45336.

10. Vercammen D et al. J Cell Biol 2007; 179: 375-380

11. Sundstrom JF et al. Nat Cell Biol 2009; 11: 1347-1354.
} 